\title{
Origin and evolution of GATA2a and GATA2b in teleosts: insights from tongue sole, Cynoglossus semilaevis
}

Jinxiang Liu, Jiajun Jiang, Zhongkai Wang, Yan He, Quanqi Zhang

Background: Following the two rounds of whole-genome duplication that occurred during deuterostome evolution, a third genome duplication occurred in the lineage of teleost fish and is considered to be responsible for much of the biological diversification within the lineage. GATA2, a member of GATA family of transcription factors, is an important regulator of gene expression in hematopoietic cell in mammals; yet the role of this gene or its putative paralogs in ray-finned fishes remains relatively unknown. Methods: In this study, we attempted to identify GATA2 sequences from the transcriptomes and genomes of multiple teleosts using the bioinformatic tools MrBayes, MEME, and PAML. Following identification, comparative analysis of genome structure, molecular evolution rate, and expression by real-time qPCR were used to predict functional divergence of GATA2 paralogs and their relative transcription in organs of female and male tongue soles (Cynoglossus semilaevis). Results: Two teleost GATA2 genes were identified in the transcriptomes of tongue sole and Japanese flounder (Paralichthysolivaceus). Synteny and phylogenetic analysis confirmed that the two genes likely originated from the teleostspecific genome duplication. Additionally, selection pressure analysis predicted these gene duplicates to have undergone purifying selection and possible divergent new functions. This was supported by differential expression pattern of GATA2a and GATA2b observed in organs of female and male tongue soles. Discussion: Our results indicate that two GATA2 genes originating from the first teleost-specific genome duplication have remained transcriptionally active in some fish species and have likely undergone neofunctionalization. This knowledge provides novel insights into the evolution of the teleost GATA2 genes and constituted important groundwork for further research on the GATA gene family. 
1 Origin and evolution of GATA2a and GATA2b in teleosts: insights from tongue sole, Cynoglossus

2 semilaevis

3 Jinxiang Liu, Jiajun Jiang, Zhongka Wang, Yan He, Quanqi Zhang

4 Key Laboratory of Marine Genetics and Breeding, Ministry of Education, Ocean University of

5 China, Qingdao, Shandong, China

6

7 Corresponding Author:

8 Quanqi Zhang

9 No 5 Yushan Road, Qingdao, Shandong, 266003, China

10 Email address: qzhang@ouc.edu.cn

11

12

13

14

15

16

17

18

19

20

21 


\section{ABSTRACT}

Background: Following the two rounds of whole-genome duplication that occurred during deuterostome evolution, a third genome duplication occurred in the lineage of teleost fish and is considered to be responsible for much of the biological diversification within the lineage. GATA2, a member of GATA family of transcription factors, is an important regulator of gene expression in hematopoietic cell in mammals; yet the role of this gene or its putative paralogs in ray-finned fishes remains relatively unknown.

Methods: In this study, we attempted to identify GATA2 sequences from the transcriptomes and genomes of multiple teleosts using the bioinformatic tools MrBayes, MEME, and PAML. Following identification, comparative analysis of genome structure, molecular evolution rate, and expression by real-time qPCR were used to predict functional divergence of GATA2 paralogs and their relative transcription in organs of female and male tongue soles (Cynoglossus semilaevis).

Results: Two teleost GATA2 genes were identified in the transcriptomes of tongue sole and Japanese flounder (Paralichthys olivaceus). Synteny and phylogenetic analysis confirmed that the two genes likely originated from the teleost-specific genome duplication. Additionally, selection pressure analysis predicted these gene duplicates to have undergone purifying selection and possible divergent new functions. This was supported by differential expression pattern of GATA2a and GATA2b observed in organs of female and male tongue soles.

Discussion: Our results indicate that two GATA2 genes originating from the first teleost-specific 
42 genome duplication have remained transcriptionally active in some fish species and have likely

43 undergone neofunctionalization. This knowledge provides novel insights into the evolution of the

44 teleost GATA2 genes and constituted important groundwork for further research on the GATA

45 gene family.

46

47

\section{INTRODUCTION}

GATA transcription factors are evolutionarily conserved proteins that bind the consensus motif WGATAR in gene regulatory regions (Evans et al. 1988; Whitelaw et al. 1990). GATA proteins are characterized by the conserved $\mathrm{N}$-terminal and $\mathrm{C}$-terminal zinc finger motifs. The $\mathrm{N}$-terminal zinc finger is required for DNA binding, whereas the C-terminal zinc finger stabilizes binding and physical interaction with other co-factors (Yang \& Evans 1992). All GATA proteins are essential to animal developmental processes, including germ layer specification, hematopoiesis, and cardiogenesis (Sorrentino et al. 2005). All the GATA family members can induce reprogramming and substitute for Oct4 (Shu et al. 2015).

GATA has been identified in vertebrates, invertebrates, fungi, and plants (Lowry \& Atchley 2000), as well as protostomes and deuterostomes (Patient \& McGhee 2002). The GATA gene family, including GATA123 and GATA456 subfamilies (Gillis et al. 2008), has undergone significant expansion after whole-genome duplication in vertebrate lineages. To date, two GATA genes have been identified in the sea urchin Strongylocentrotus purpuratus, and two in the hemichordate Saccoglossus kowalevskii, the urochordate Ciona intestinalis, and the cephalochordate Branchiostoma floridae. Meanwhile, six GATA transcription factors (GATA1 to GATA6) have been found in tetrapods and teleosts (Gillis et al. 2009). 
63 Previous studies have verified multiple rounds of whole-genome duplication in vertebrate lineages,

64 which may play a significant role in vertebrate evolution (Hoegg \& Meyer 2005; Hoffmann et al.

65 2012; Hughes 1999). Interestingly, a third whole-genome duplication event (3R) occurred in

66 teleosts (Amores et al. 1998; Postlethwait et al. 1998). Teleost-specific genome duplication (TGD)

67 provided more gene copies, contributing to the evolutionary and phenotypic diversification of

teleosts. TGD-derived gene duplicates supported the cause-effect relationship between gene copy

number and species diversity (Siegel et al. 2007). The duplicated genes might possess great

divergence from their ancestors, as demonstrated by the changes in evolutionary rates, expression

patterns, and regulatory mechanisms observed across the teleost lineage (Braasch et al. 2006;

Hoegg \& Meyer 2007; Mulley et al. 2006). Duplicated genes have three main fates, that is,

nonfunctionalization, subfunctionalization, and neofunctionalization (Force et al. 1999).

In teleosts, research investigating GATA2 has been minimal. To better understand the origination

and functional divergence of GATA2 in teleost, this study aimed to investigate GATA2 gene(s)

from the transcriptome of tongue sole, Japanese flounder, and other teleosts. Following

identification of two GATA2 genes in the tongue sole, chromosomal synteny and phylogenetic

analysis of these genes was performed to investigate the origin and evolution of GATA2 in teleosts.

Then, analysis of genomic structure, molecular positive selection, and expression pattern of the

two GATA2 genes in tongue sole were performed to identify potential changes in functionality for

the duplicated GATA2 genes within the teleost lineage. This study provides evidence to support the GATA family expansion theory that the increase of GATA members follows the wholegenome duplication. It also lays the foundation for further evolutionary and functional studies of 
84 the GATA gene family in teleosts.

85

86

87

88

\section{MATERIALS AND METHODS}

\section{Ethics Statement}

All research was conducted in accordance with the Institutional Animal Care and Use Committee of the Ocean University of China and with the China Government Principles for the Utilization and Care of Vertebrate Animals Used in Testing, Research, and Training (State science and technology commission of the People's Republic of China for No. 2, October 31, 1988. http://www.gov.cn/gongbao/content/2011/content_1860757.htm).

\section{Fish}

Healthy tongue sole (three females and three males) of one-year-old were chosen from a larger cohort population. The fish were anesthetized (MS-222 at $30 \mu \mathrm{g} / \mathrm{mL})$ and then killed by severing spinal cord. Brain, heart, intestine, kidney, liver, spleen, and gonad organs were collected in triplicate from each fish. All of the samples were immediately frozen using liquid nitrogen and stored at $-80^{\circ} \mathrm{C}$ for total RNA extraction.

\section{Identification of GATA gene family sequences in the tongue sole}

GATA gene family members were identified from Amazon molly (Poecilia formosa), fugu (Takifugu rubripes), medaka (Oryzias latipes), stickleback (Gasterosteus aculeatus), tetraodon (Tetraodon nigroviridis), and tilapia (Oreochromis niloticus) whose genomes are completely sequenced and available from the Ensembl database. The retrieved sequences were used as query sequences in BLAST searches. The mRNA sequences of GATA genes were identified using tBLASTn analysis from the tongue sole transcriptome previously sequenced by our laboratory. 
105 The transcriptome was generated from a total of 749,954 reads using a single 454 sequencing run 106 and assembled into 62,632 contigs, of which 26,589 sequences were successfully annotated (Wang 107 et al. 2014b). These fragments were used to search for the corresponding chromosomal regions 108 containing in the tongue sole genome from NCBI (GenBank accession: PRJNA73987). CsGATA2a was found on scaffold385_11, and CsGATA2b was identified on scaffold57_8.

\section{Identification of GATA gene family sequences in the Japanese flounder}

The sequences retrieved from tongue sole and the other six teleosts listed above were used as query sequences to search for PoGATA genes. The sequences were identified from the Japanese flounder transcriptome through tBLASTn analysis (Wang et al. 2014a). An unpublished Japanese flounder genome was used to search for the DNA sequences of PoGATA2a and PoGATA2b (Supplemental seq file).

\section{GATA2 sequence alignment and phylogenetic analysis}

117 The sequence alignments of GATA2a and GATA2b were based on their predicted peptide sequences using Clustal X with default parameters (Chenna et al. 2003). Phylogenetic trees were constructed to confirm the ortholog and paralog relationships of both duplicates. The sequences used to construct gene trees were retrieved from Ensembl and NCBI (species names, gene names, and accession numbers are available in Table S1). The most appropriate substitution model of molecular evolution was determined using JModelTest v2.1.4 (Darriba et al. 2012). To confirm the tree topologies, a Bayesian tree and a maximum likelihood tree were respectively constructed using MrBayes v3.2.2 (Huelsenbeck \& Ronquist 2001; Ronquist et al. 2012) and phyML v3.1

(Guindon et al. 2010). MrBayes was run for 400,000 generations with two runs and four chains in 
parallel and a burn-in of 25\%. PhyML was run for 1000 replications. Other parameters were based

127

128

129

130

131

132

133

134

135

136

137

138

139

140

141

142

143

144

145

146

on the result of JModelTest.

\section{Tests for positive selection in GATA2a and GATA2b}

A Bayesian tree was constructed using MrBayes based on GATA2a and GATA2b. The tree includes all species used for positive selection analyses (Table S1). The TIM3+I+G model with base frequencies and substitution rate matrix estimated from the parameters (as suggested by

JModelTest) was used. The standard site model in CODEML of PAML v4.7 was used to calculate selection pressures (Yang 2007). The site model employed ML estimation of the ratio of nonsynonymous to synonymous substitutions $\left(\mathrm{d}_{\mathrm{N}} / \mathrm{d}_{\mathrm{S}=} \omega\right)$ and nested likelihood ratio tests (LRTs) on a phylogeny tree.

\section{Genomic structure, motif, and synteny analysis of teleost GATA2 paralogs}

Diagrams of exon-intron structures were obtained using the online Gene Structure Display Server 2.0 (GSDS: http://gsds.cbi.pku.edu.cn) with CDS and genomic sequences (Hu et al. 2015). Motifs in the candidate GATA2 DNA sequences were identified using MEME (Bailey et al. 2009). The Synteny Database (Catchen et al. 2009) was used to generate dotplots of the human GATA2 gene region on chromosome Hsa3 and the genome of zebrafish to analyze the syntenic conservation between fish and human chromosomes.

\section{RNA isolation, cDNA synthesis, and qRT-PCR}

Total RNA was extracted from organ samples with Trizol reagent (Invitrogen, Carlsbad, CA, USA) in accordance with the manufacturer's instructions. DNA was removed using DNase I (TaKaRa, Dalian, China) treated with $2 \mathrm{~h}$ at $37^{\circ} \mathrm{C}$, and the protein was digested using an RNAclean 
147 RNA Kit (Biomed, Beijing, China). The quality and quantity of the extracted RNA were identified

148 via electrophoresis and Nanophotometer® Pearl (Implen GmbH, Munich, Germany). Frist-strand

149 cDNA was synthesized using the PrimeScript ${ }^{\mathrm{TM}}$ RT-PCR Kit (TaKaRa) in accordance with the

150 manufacturer's instructions.

151 Quantitative Real-time was conducted on a LightCycler 480 (Roche, Forrentrasse, Switzerland).

152 The respective primer pairs for GATA2a and GATA2b were Cs-GATA2a-RT and Cs-GATA2b-

153 RT (Table S2), which were designed by IDT (http://www.idtdna.com/Primerquest/Home/Index)

154 in the 3' UTR of both genes. Standard curves were established from a serial dilution of plasmids

155 containing GATA2a, GATA2b, and reference gene RPL17 fragments. Efficiency values (91.58\%,

$15688.25 \%$, and $92.10 \%$, respectively) were calculated by standard curves (Boyle et al, 2009). cDNA

157 from three females and males were diluted as templates $(10 \mathrm{ng} / \mu \mathrm{L})$ for sample assessment. The

SYBR Green master mix (Roche, Switzerland) was used as the PCR detection system. Three male

and three female individuals were collected. The same organ from three male or three female individuals were pooled as one sample for expression analysis, and the experiments for each pooled sample were performed in triplicate. Thermocycling consisted of an initial polymerase activation of $30 \mathrm{~s}$ at $94{ }^{\circ} \mathrm{C}$, followed by 40 cycles at $94{ }^{\circ} \mathrm{C}$ for $15 \mathrm{~s}$ and $60{ }^{\circ} \mathrm{C}$ for $45 \mathrm{~s}$. Product specificity was ensured through melting curve analysis which consisted of 40 cycles. RPL17 of tongue sole was used as the reference gene to normalize the expression which has been shown to be stably expressed between male and female tongue soles in multiple organ types (Liu et al. 2014). The sizes of GATA2a, GATA2b, and RPL17 amplicons were 121bp, 122bp, and 114bp, and melt curve starting temperatures were $60^{\circ} \mathrm{C}, 61^{\circ} \mathrm{C}$, and $60^{\circ} \mathrm{C}$, respectively. Data were analyzed 
168 through the $2^{-\Delta \Delta \mathrm{Ct}}$ method.

\section{Statistical analysis}

170 qRT-PCR data were statistically analyzed using one-way ANOVA on log10-transformed data 171 followed by LSD test using SPSS 20.0, and $P<0.05$ was considered to indicate statistical 172 significance. All data were expressed as mean \pm standard error of the mean (SEM).

\section{RESULTS}

\section{Identification of GATA genes}

175 We identified GATA1, GATA2a, GATA2b, GATA3, GATA4, GATA5, and GATA6 from the transcriptomes of tongue sole and Japanese flounder via tBLAST to infer the origin and evolutionary history of the GATA gene family in teleosts. Other GATA genes were searched from Ensembl and NCBI. The GATA family can be divided into the GATA123 and GATA456 subfamilies. Protein analysis showed that the GATA family in teleosts comprised two conserved zinc finger motifs at the $\mathrm{N}$-terminal and the $\mathrm{C}$-terminal domains. However, the different GATA paralogs in teleosts had varied lengths. Seven GATA genes, including two GATA2 genes (GATA2a and GATA2b), were detected in the teleost GATA family. Only six GATA genes were detected in tetrapods.

\section{Phylogenetic relationships and evolution of the GATA gene family}

The identified DNA sequences were analyzed to investigate the evolutionary relationship of GATA genes among various teleosts using multiple sequence alignment with Clustal X. A phylogenetic tree of the GATA gene family was constructed using MrBayes and phyML based on 
188 189 190

the alignment results. The two programs inferred similar topologies, which indicated that the GATA gene family could be divided into seven well-conserved clades and two subfamilies in teleosts (Figure 1).

Our results also indicated distinct ancestral relationship within each subfamily of the GATA gene family. A close relationship was observed between GATA2a/b and GATA3 within the GATA123 subfamily, and between GATA5 and GATA6 within the GATA456 subfamily.

\section{Phylogenetic analysis of teleost-specific GATA2a and GATA2b}

Multiple amino acid alignment was conducted to explore the origin, generation, and differentiation of GATA2 $a$ and GATA2 $b$ in teleosts. The sequence similarity between GATA2a and GATA2b was $82.55 \%$, with two highly conserved zinc finger motifs. Sequence alignment suggested the occurrence of two GATA2b-specific mutations in the N-terminal and C-terminal zinc fingers. Specifically, serine was dehydroxymethyled into glycine in the N-terminal zinc finger motif, and alanine was demethylated into glycine in the C-terminal zinc finger motif (Figure 2 and Figure S1). The N-terminal zinc finger motif can stabilize the binding and physically interact with other co-factors, and the C-terminal zinc finger motif is required for DNA binding. Thus the dehydroxymethylation and demethylation mutations might trigger protein structure alteration, and further affect the molecular functions in biological processes.

The phylogenetic trees of GATA2a and GATA2b in teleosts were constructed using MrBayes and phyML, with the human GATA2 sequence as an outgroup. The two trees were similar in topology with minimal bootstrap differences. Results indicated that teleost GATA2 genes could be divided into two well-conserved clusters: GATA2a and GATA2b (Figure 3), implying that GATA2a and 
209 GATA2b in teleosts were probably generated from the same ancestor.

\section{Genomic structures of teleost GATA2}

211 Gene structure graphics were constructed by the online program Gene Structure Display Server to 212 analyze the evolutionary mechanism of GATA2. The graphics showed that both GATA2a and 213 GATA2b had five exons in CDS, except for PfGATA2b, which had an extra intron dividing the 214 second exon into two segments. The lengths of each corresponding exon were highly conserved, 215 but intron lengths varied among species. The GATA2a and GATA2b in fugu and tetraodon had 216 the shortest intron lengths. In most teleosts, the GATA2b gene was longer than the GATA2a gene, 217 which might infer that the two subtypes of GATA2 had undergone gene differentiation, that is, they originated from a common ancestor but diverged into two genes differing in protein structure and functions (Figure S2A). This inference was further supported by motif prediction on teleost GATA2 by MEME. Four main motifs (motifs 1, 2, 3, and 4) were predicted in both GATA2a and GATA2b. An additional motif 4 was predicted at the end of GATA2b in most teleosts (Figure S2B).

\section{Synteny analysis of teleost GATA2 paralogs}

224 Chromosomal synteny analysis was carried out between human and zebrafish to test whether that GATA2 paralogs originated from whole-genome duplication. Conserved synteny dotplots showed that the GATA2a and GATA2b regions in zebrafish shared conserved synteny. The zebrafish GATA2a region on Dre11 shared conserved synteny neither with the zebrafish GATA2b region Dre6 nor with the human GATA2 region Hsa3 (Figure 4). Previous studies confirmed that these chromosomes originated from the common ancestral chromosome and duplicated during the 
230

231

232

233

234

235

236

237

238

239

240

241

242

243

244

245

246

247

248

249

250

teleost-specific genome duplication (Kasahara et al. 2007; Nakatani et al. 2007).

Gene neighborhood analysis showed highly conserved synteny within GATA2a or GATA2b and

between the two genes. In teleosts, the genes near GATA2a, except for some genes lost in tetraodon duplication era, were mostly conserved and shared the same direction (Figure 5A). Long

fragments consisting of several genes were lost in the upstream and downstream regions of GATA2b in Amazon molly and fugu, but the other genes remained conserved. Comparison of the upstream genes of GATA2a and GATA2b revealed that a fragment including four genes was conserved, albeit in opposite directions (indicated by blank pentagons) (Figure 5B). A gene in the upstream region and a two-gene string in the downstream region (indicated by blank pentagons) were also highly conserved between GATA2a and GATA2b. These results implied that the genes neighboring teleost GATA2a or GATA2b were highly conserved, and more conserved among teleosts after duplication.

\section{Molecular evolution of teleost GATA2a and GATA2b}

In general, phenotypic differences can arise from mutations affecting protein functions or changes in gene regulation (Stainier et al. 1996). Therefore, we examined the coding sequence evolution in two GATA2 paralogs to test for positive selection and potential functional changes in teleosts. The site models in PAML were used to assess different selective pressures. The estimation of positive selection based on the phylogenetic trees is shown in Figure S3. Three model pairs (M0/M3, M1a/M2a, and M7/M8) were selected and compared with the site-specific codeml model to test whether variable $\omega$ ratios occurred at amino acid sites. The parameters and the LRT results are listed in Table 1. 
251

252

253

254

255

256

257

258

259

260

261

262

263

264

265

266

267

268

269

270

In GATA2, M3 (discrete) was significantly better than M0 (one-ratio) $(P<0.05)$. Thus, M0 was rejected, indicating the extreme variation in selection pressure among amino acid sites. Overall, the GATA2 sequences had undergone positive selection. Additional tests with M1a (neutral) and M2a (selection), M7 (beta)/M8 (beta \& $\omega$ ) and M8a were conducted using the chi2 program in PAML. The LRT significantly differed in the M7/M8 pair of GATA2b $(P<0.05)$. One candidate amino acid site for positive selection $(356 \mathrm{P}, P<0.05)$ was identified $\left(356 \mathrm{P}^{*}\right)$ through the Bayes Empirical Bayes (BEB) method of M8. No site under positive selection was identified in GATA2a. Then, the relationship between amino acid sites under positive selection and function divergence was analyzed. The site $356 \mathrm{P}$ with a posterior probability $>0.95$ was located in the C-terminal zinc finger in GATA2b, indicating that GATA2b, especially its motif, had experienced a strong selective pressure, which might develop mechanism adapting to water environment.

\section{Expression levels of GATA2a and GATA2b in organ}

Quantitative real-time PCR using RNA extracted from multiple tongue sole organs was performed to test if transcription regulation of GATA2a and GATA2b had undergone divergence in teleosts.

Both genes were expressed in all organs tested but possessed distinct levels of expression. Heart and the brain showed higher relative expression of GATA2a/b than other somatic organs in both sexes, and extraordinarily high GATA2b expression was found in the heart (Figure S4). A sexual dimorphic expression pattern was observed in the gonads. In the ovary, GATA2a expression was hardly observed and GATA2b expression was very low (Figure 6A), while in the testis, GATA2a expression was moderate and GATA2b expression was relatively high (Figure 6B). 
DISCUSSION

272

273

274

275

\section{Expansion of vertebrate GATA transcription factor genes during multiple whole-genome}

\section{duplications}

GATA transcription factors play crucial roles in regulating the development and differentiation processes including hematopoiesis, cardiogenesis, and germ layer specification (Holtzinger \& Evans 2005; LaVoie 2003). In the present study, seven GATA genes were identified from both tongue sole and Japanese flounder transcriptomes. Indeed, all teleosts analyzed in this study possessed seven GATA genes, including six GATA genes shared with tetrapods and an additional teleost-specific GATA2 duplication. As teleosts have undergone a unique 3R genome duplication, some gene families became larger in teleosts than in tetrapods or chondrichthyes. Thus, the present results are consistent with previous reports that GATA gene family expansion occurred through genome duplication and that clade-specific conserved losses of duplicated paralogs occurred after duplication (Gillis et al. 2009).

Phylogenetic analysis suggested that the GATA gene family had undergone distinct expansion that separated the GATA123 and GATA456 subfamilies, both of which were subsequently expanded.

Our results differed from findings on the evolution of the GATA gene family in protostomes but agreed with those on the evolution of vertebrates. In protostomes, only the GATA456 subfamily appeared to have undergone expansion (Gillis et al. 2008). By contrast, the GATA123 and GATA456 subfamilies both expanded in deuterostomes through the retention of duplicated GATA genes during multiple whole-genome duplications (Dehal \& Boore 2005). Our molecular phylogenetic analysis, together with the conserved syntenic paralogs (Gillis et al. 2009), provided 
292 evidence to support the expansion through genome duplication.

\section{Origin of GATA2 paralogs}

294 Several molecular mechanisms, such as gene duplication, exon shuffling, gene fission and fusion, retrotranspositon, and mobile elements, have been proposed to understand the origin of new genes

(Long et al. 2003). Gene duplication events, including single-gene duplication, segmental duplication, and genome duplication, are crucial to produce new genes (Bailey et al. 2002;

Samonte \& Eichler 2002). In the present study, the results of chromosomal synteny analysis and gene-neighborhood synteny analysis indicated that the two GATA2 paralogs were generated through genome duplication in teleosts.

The fate of newborn genes is diverse. Some scholars believed that a number of a duplicate gene pairs eventually become nonfunctional and that most duplicates eventually perish as pseudogenes (Bailey et al. 1978). Gene duplicates possibly acquire new functions (neofunctionalization) or undergo subfunctionalization and are preserved in a lineage (Force et al. 1999; Kimura \& King 1979; Li 1980). During whole-genome duplication of yeast, arabidopsis, rice and tetraodon, all of the genes were duplicated, but only $10 \%-30 \%$ of new genes were preserved, and others were lost in evolution (Byrne \& Wolfe 2005; Paterson et al. 2006). In the present study, ohnolog-gonemissing (ogm) was observed throughout the evolution of the GATA gene. Based on phylogenetic analysis, we conjectured that GATA1-ogm and GATA4-ogm occurred after 2R, which is consistent with a former study (Gillis et al. 2009). Most GATA paralogs, except GATA2, were lost after 3R in teleosts. GATA2 may have been preserved in the evolutionary process because of environmental pressure and further supports that the two GATA2 paralogs originated from 3R 
313 duplication.

\section{Structures of the GATA2a and GATA2b genes}

315 The structure of GATA genes is generally conserved, as shown in protostomes and deuterostomes

316 relative to vertebrate transcriptomes (Gillis et al. 2008; Gillis et al. 2009). In the present study, we

317 examined the conservation of the exon/intron structures of GATA2a and GATA2b in teleosts. The

318 genomic structure of GATA2 was conserved; all GATA2 genes, except for PfGATA2b, contained

319 five exons in CDS. The lengths of the five exons were conserved, but the lengths of the introns

varied. Introns are important indicators in eukaryotic evolution, where the gain and loss of introns

reflect positive correlation or negative correlation with the coding-sequence evolution rate (Carmel

et al. 2007; Slamovits \& Keeling 2009). The intron lengths of GATA2a were generally shorter

than those of GATA2b, suggesting that the two GATA2 genes had diverged. Meanwhile, motif

prediction showed an additional motif 4 in GATA2b. This motif might separate GATA2b from

GATA2a functionally, which was consistent with the phylogenetic results. These results implied

that GATA2a and GATA2b in teleosts separated from each other and generated different structures

and functions. We inferred that the sequence of GATA2a and GATA2b had been changed under

selection pressure.

\section{Potential for functional divergence of GATA2a and GATA2b}

In general, new genes evolve with rapid changes in their sequence and structure (Wang et al. 2002;

Zhang et al. 2002), and mutation is the initial condition in evolution. Positive Darwinian selection

may be another important force driving the evolution of new genes (Ohta 1994; Walsh 1995). The

evolutionary rates of gene pairs that originated from duplication are usually different, and the rapid 
334 evolution of one of the gene pairs is a general phenomenon (Johnson et al. 2001; Wang et al. 335 2002). In the present study, the teleost GATA2 phylogenetic tree provided evidence that the 336 evolutionary rate of GATA2b was faster than that of GATA2a under current environmental 337 pressure. Thus, GATA2b has likely diverged from an ancestral GATA2 more similar to present 338 GATA2a paralog.

339 The amino acid sequences of GATA genes contain the well-conserved N-terminal and C-terminal 340 zinc finger motifs, which significantly contribute to structure and function. In the present study, 341 the two zinc finger motifs were highly conserved in GATA2a and GATA2b in teleosts. Two 342 mutated amino acid sites were found located in the two zinc finger motifs in GATA2b relative to 343 GATA2a. Measuring the rate of relaxation and determining the presence of amino acid residue under positive selection are crucial to determine whether positive selection has driven the evolution of the GATA2 paralogs and whether or not selection constraints affect GATA2 genes after duplication in teleosts. The results of selection pressure analysis provided evidence of purifying selection, and one site (356P) in GATA2b was predicted to have undergone a strong positive selection. Interestingly, this site was located in the C-terminal zinc finger motif, which has been inferred to play an important role during evolution. Therefore, this positively selected site might affect the binding activity of GATA2b or even affect the selection of binding sites, resulting in the functional divergence between GATA2a and GATA2b.

In the current study, transcriptional analysis was performed using qRT-PCR. We focused on the overall expression pattern but not the individual differences, so pooled samples were used. Three experimental repeats for each pooled sample were performed to ensure the operational accuracy 
355

356

357

358

359

360

361

362

363

364

365

366

367

368

369

370

371

372

373

374

375

and the results could effectively reflect the actual expression levels on average. The expression patterns of GATA2a and GATA2b were similar in most somatic organs, but sexual dimorphic expression was apparent, especially in the spleen and the gonad. Previous studies have shown that new genes have evolved in conjunction with rapid changes in expression (Wang et al. 2002; Zhang et al. 2002), and the differential expression of these genes was believed to be the first step in functional divergence. The classical model for the evolution of duplicate genes identifies two possibilities: one is that one of the duplicated genes degenerates by accumulating deleterious mutations; the other is that one duplicate acquires a new adaptive function (Ohno 1970). However, the duplication-degeneration-complementation (DDC) model predicts that the duplicate gene preservation involves the partitioning of ancestral functions rather than the evolution of new functions (Force et al. 1999). Moreover, the expression levels of GATA2b in the brain, the pituitary gland, and the gonad differed between females and males in tilapia (Zhang 2009). Based on the results of our present study, we hypothesize that the differential transcription of the GATA2 paralogs in tongue sole follow the DDC model; that is, GATA2a and GATA2b partitioned the ancestral functions of GATA2 in teleosts. GATA2a might have maintained the functions of GATA2 in hemopoiesis and in the multiplication and differentiation of hematopoietic stem cells, whereas GATA2b might have acquired some functions related to sexual differentiation and gonad development or sexual maturation. These results provide preliminary evidence that the duplicated GATA2 genes may have undergone neofunctionalization in teleosts.

\section{CONCLUSIONS}

In summary, we investigate the origin of teleost GATA2a/b genes and reports for the first time 
376

377

378

379

380

381

382

383

384

385

386

387

388

389

390

391

392

393

394

395

that two GATA2 genes are present in teleosts as a result of TGD. In addition, our results indicate

possible neofunctionalization of the duplicated GATA2 genes, providing novel insight into the teleost GATA gene family and future functional studies of GATA2 in fish.

\section{REFERENCES}

Amores A, Force A, Yan YL, Joly L, Amemiya C, Fritz A, Ho RK, Langeland J, Prince V, Wang YL, Westerfield M, Ekker M, Postlethwait JH. 1998. Zebrafish hox clusters and vertebrate genome evolution. Science 282:1711-1714.

Bailey GS, Poulter RT, Stockwell PA. 1978. Gene duplication in tetraploid fish: model for gene silencing at unlinked duplicated loci. Proceedings of the National Academy of Sciences of the United States of America 75:5575-5579.

Bailey JA, Gu Z, Clark RA, Reinert K, Samonte RV, Schwartz S, Adams MD, Myers EW, Li PW, Eichler EE. 2002. Recent segmental duplications in the human genome. Science 297:10031007.

Bailey TL, Boden M, Buske FA, Frith M, Grant CE, Clementi L, Ren J, Li WW, Noble WS. 2009. MEME Suite: tools for motif discovery and searching. Nucleic Acids Research 37:W202W208.

Boyle B, Dallaire N, MacKay J. 2009. Evaluation of the impact of single nucleotide polymorphisms and primer mismatches on quantitative PCR. BMC Biotechnology 9:75-75.

Braasch I, Salzburger W, Meyer A. 2006. Asymmetric evolution in two fish-specifically duplicated receptor tyrosine kinase paralogons involved in teleost coloration. Molecular 
Biology and Evolution 23:1192-1202.

397

398

399

400

401

402

403

404

405

406

407

408

409

410

411

412

413

414

415

416

Byrne KP, Wolfe KH. 2005. The Yeast Gene Order Browser: Combining curated homology and syntenic context reveals gene fate in polyploid species. Genome Research 15:1456-1461.

Carmel L, Rogozin IB, Wolf YI, Koonin EV. 2007. Evolutionarily conserved genes preferentially accumulate introns. Genome Research 17:1045-1050.

Catchen JM, Conery JS, Postlethwait JH. 2009. Automated identification of conserved synteny after whole-genome duplication. Genome Research 19:1497-1505.

Chenna R, Sugawara H, Koike T, Lopez R, Gibson TJ, Higgins DG, Thompson JD. 2003. Multiple sequence alignment with the Clustal series of programs. Nucleic Acids Research 31:34973500.

Darriba D, Taboada GL, Doallo R, Posada D. 2012. jModelTest 2: more models, new heuristics and parallel computing. Nature Methods 9:772-772.

Dehal P, Boore JL. 2005. Two rounds of whole genome duplication in the ancestral vertebrate. PLoS Biology 3:e314.

Evans T, Reitman M, Felsenfeld G. 1988. An erythrocyte-specific DNA-binding factor recognizes a regulatory sequence common to all chicken globin genes. Proceedings of the National Academy of Sciences of the United States of America 85:5976-5980.

Force A, Lynch M, Pickett FB, Amores A, Yan YL, Postlethwait J. 1999. Preservation of duplicate genes by complementary, degenerative mutations. Genetics 151:1531-1545.

Gillis WQ, Bowerman BF, Schneider SQ. 2008. The evolution of protostome GATA factors: molecular phylogenetics, synteny, and intron/exon structure reveal orthologous 
relationships. BMC Evolutionary Biology 8:1-15.

418 Gillis WQ, St John J, Bowerman B, Schneider SQ. 2009. Whole genome duplications and expansion of the vertebrate GATA transcription factor gene family. BMC Evolutionary Biology 9:207-207.

Guindon S, Dufayard JF, Lefort V, Anisimova M, Hordijk W, Gascuel O. 2010. New algorithms and methods to estimate maximum-likelihood phylogenies: Assessing the performance of PhyML 3.0. Systematic Biology 59:307-321.

Hoegg S, Meyer A. 2005. Hox clusters as models for vertebrate genome evolution. Trends in Genetics 21:421-424.

Hoegg S, Meyer A. 2007. Phylogenomic analyses of KCNA gene clusters in vertebrates: why do gene clusters stay intact? BMC Evolutionary Biology 7:139-139.

Hoffmann FG, Opazo JC, Storz JF. 2012. Whole-genome duplications spurred the functional diversification of the globin gene superfamily in vertebrates. Molecular Biology and Evolution 29:303-312.

Holtzinger A, Evans T. 2005. Gata4 regulates the formation of multiple organs. Development

Hu B, Jin J, Guo AY, Zhang H, Luo J, Gao G. 2015. GSDS 2.0: an upgraded gene feature visualization server. Bioinformatics 31:1296-1297.

Huelsenbeck JP, Ronquist F. 2001. MRBAYES: Bayesian inference of phylogenetic trees. Bioinformatics 17:754-755. 
hypothesis of two rounds of genome duplication early in vertebrate history. Journal of Molecular Evolution 48:565-576.

Johnson ME, Viggiano L, Bailey JA, Abdul-Rauf M, Goodwin G, Rocchi M, Eichler EE. 2001. Positive selection of a gene family during the emergence of humans and African apes. Nature 413:514-519.

Kasahara M, Naruse K, Sasaki S, Nakatani Y, Qu W, Ahsan B, Yamada T, Nagayasu Y, Doi K, Kasai Y, Jindo T, Kobayashi D, Shimada A, Toyoda A, Kuroki Y, Fujiyama A, Sasaki T, Shimizu A, Asakawa S, Shimizu N, Hashimoto S-i, Yang J, Lee Y, Matsushima K, Sugano S, Sakaizumi M, Narita T, Ohishi K, Haga S, Ohta F, Nomoto H, Nogata K, Morishita T, Endo T, Shin-I T, Takeda H, Morishita S, Kohara Y. 2007. The medaka draft genome and insights into vertebrate genome evolution. Nature 447:714-719.

Kimura M, King JL. 1979. Fixation of a deleterious allele at one of two "duplicate" loci by mutation pressure and random drift. Proceedings of the National Academy of Sciences of the United States of America 76:2858-2861.

LaVoie HA. 2003. The role of GATA in mammalian reproduction. Experimental Biology and Medicine 228:1282-1290.

Li WH. 1980. Rate of gene silencing at duplicate loci: A theoretical study and interpretation of data from tetraploid fishes. Genetics 95:237-258.

Liu C, Xin N, Zhai Y, Jiang L, Zhai J, Zhang Q, Qi J. 2014. Reference gene selection for quantitative real-time RT-PCR normalization in the half-smooth tongue sole (Cynoglossus semilaevis) at different developmental stages, in various tissue types and on exposure to 
459

460

461

462

463

464

465

466

467

468

469

470

471

472

473

474

475

476

477

478

479

chemicals. PLoS ONE 9:e91715.

Long M, Betran E, Thornton K, Wang W. 2003. The origin of new genes: glimpses from the young and old. Nature Reviews Genetics 4:865-875.

Lowry JA, Atchley WR. 2000. Molecular evolution of the GATA family of transcription factors: Conservation within the DNA-Binding domain. Journal of Molecular Evolution 50:103115.

Mulley JF, Chiu CH, Holland PWH. 2006. Breakup of a homeobox cluster after genome duplication in teleosts. Proceedings of the National Academy of Sciences of the United States of America 103:10369-10372.

Nakatani Y, Takeda H, Kohara Y, Morishita S. 2007. Reconstruction of the vertebrate ancestral genome reveals dynamic genome reorganization in early vertebrates. Genome Research $17: 1254-1265$.

Ohno S. 1970. Evolution by gene duplication. Springer-Verlag, New York.

Ohta T. 1994. Further examples of evolution by gene duplication revealed through DNA sequence comparisons. Genetics 138:1331-1337.

Paterson AH, Chapman BA, Kissinger JC, Bowers JE, Feltus FA, Estill JC. 2006. Many gene and domain families have convergent fates following independent whole-genome duplication events in Arabidopsis, Oryza, Saccharomyces and Tetraodon. Trends in Genetics 22:597602.

Patient RK, McGhee JD. 2002. The GATA family (vertebrates and invertebrates). Current Opinion in Genetics \& Development 12:416-422. 
480

481

482

483

484

485

486

487

488

489

490

491

492

493

494

495

496

497

498

499

500

Postlethwait JH, Yan YL, Gates MA, Horne S, Amores A, Brownlie A, Donovan A, Donovan A, Egan ES, Force A, Gong Z, Goutel C, Fritz A, Kelsh R, Knapik E, Liao E, Paw B, Ransom D, Singer A, Thomson M, Abduljabbar TS, Yelick P, Beier D, Joly JS, Larhammar D, Rosa F, Westerfield M, Zon LI, Johnson SL, Talbot WS. 1998. Vertebrate genome evolution and the zebrafish gene map. Nature Genetics 19:303-303.

Ronquist F, Teslenko M, van der Mark P, Ayres DL, Darling A, Höhna S, Larget B, Liu L, Suchard MA, Huelsenbeck JP. 2012. MrBayes 3.2: Efficient Bayesian phylogenetic inference and model choice across a large model space. Systematic Biology 61:539-542.

Samonte RV, Eichler EE. 2002. Segmental duplications and the evolution of the primate genome. Nature Reviews Genetics 3:65-72.

Shu J, Zhang K, Zhang M, Yao A, Shao S, Du F, Yang C, Chen W, Wu C, Yang W, Sun Y, Deng H. 2015. GATA family members as inducers for cellular reprogramming to pluripotency. Cell Reseach 25:169-180.

Siegel N, Hoegg S, Salzburger W, Braasch I, Meyer A. 2007. Comparative genomics of ParaHox clusters of teleost fishes: gene cluster breakup and the retention of gene sets following whole genome duplications. BMC Genomics 8:312-312.

Slamovits CH, Keeling PJ. 2009. Evolution of ultrasmall spliceosomal introns in highly reduced nuclear genomes. Molecular Biology and Evolution 26:1699-1705.

Sorrentino RP, Gajewski KM, Schulz RA. 2005. GATA factors in Drosophila heart and blood cell development. Seminars in Cell \& Developmental Biology 16:107-116.

Stainier DY, Fouquet B, Chen JN, Warren KS, Weinstein BM, Meiler SE, Mohideen MA, 
501

502

503

504

505

506

507

508

509

510

511

512

513

514

515

516

517

518

519

520

521

Neuhauss SC, Solnica-Krezel L, Schier AF, Zwartkruis F, Stemple DL, Malicki J, Driever W, Fishman MC. 1996. Mutations affecting the formation and function of the cardiovascular system in the zebrafish embryo. Development 123:285-292.

Walsh JB. 1995. How often do duplicated genes evolve new functions? Genetics 139:421-428.

Wang W, Brunet FG, Nevo E, Long M. 2002. Origin of sphinx, a young chimeric RNA gene in Drosophila melanogaster. Proceedings of the National Academy of Sciences of the United States of America 99:4448-4453.

Wang W, Wang J, You F, Ma L, Yang X, Gao J, He Y, Qi J, Yu H, Wang Z, Zhang Q . 2014a. Detection of alternative splice and gene duplication by RNA sequencing in Japanese flounder, Paralichthys olivaceus. G3: Genes|Genomes|Genetics 4:2419-2424.

Wang W, Yi Q, Ma L, Zhou X, Zhao H, Wang X, Qi J, Yu H, Wang Z, Zhang Q. 2014 b. Sequencing and characterization of the transcriptome of half-smooth tongue sole (Cynoglossus semilaevis). BMC Genomics 15:470.

Whitelaw E, Tsai SF, Hogben P, Orkin SH. 1990. Regulated expression of globin chains and the erythroid transcription factor GATA-1 during erythropoiesis in the developing mouse. Molecular and Cellular Biology 10:6596-6606.

Yang HY, Evans T. 1992. Distinct roles for the two cGATA-1 finger domains. Molecular and Cellular Biology 12:4562-4570.

Yang Z. 2007. PAML 4: Phylogenetic analysis by maximum likelihood. Molecular Biology and Evolution 24:1586-1591.

Zhang J, Zhang YP, Rosenberg HF. 2002. Adaptive evolution of a duplicated pancreatic 
523 Zhang W. 2009. Molecular cloning, gene expression of GATA-2, p450(11 $\beta$ ) and their possible roles in gonadal differentiation and gametogenesis in tilapia. M. Phil. Thesis, Southwest University.

526

527

FIGURE LEGENDS

528

Figure 1 Phylogenetic analyses of vertebrate GATA gene family. Phylogenetic tree constructed using MrBayes with the TPM1uf $+\mathrm{I}+\mathrm{G}$ model; $\mathrm{MCMC}=400,000$ generations. Values at the tree nodes represent posterior probabilities. On-Oreochromis niloticus, Cs-Cynoglossus semilaevis,

Tn-Tetraodon nigroviridis, Mm-Mus musculus, Gg-Gallus gallus; Hs-Homo sapiens; Nv-

Nematostella vectensis.

Figure 2 Partial multiple sequence alignment of the deduced GATA2a and GATA2b protein sequences. In teleosts, two conserved zinc finger motifs were found in GATA2a and GATA2b (underlined sequences). Two GATA2b-specific mutations were identified in zinc finger motifs

537 (star-shaped site). The proline site (arrowhead) in the N-terminal zinc finger motif had undergone positive selection.

Figure 3 Phylogenetic analysis of teleost GATA2. (A) Phylogenetic tree constructed based on

541 GATA2 $\mathrm{a}$ and GATA2b in teleosts by using MrBayes with the TIM3+I+G model; MCMC =

542 400,000. (B) Maximum likelihood phylogenetic tree constructed using phyML with the 
543 TIM3 $+\mathrm{I}+\mathrm{G}$ model. PhyML was run for 1000 replications. Numbers at the nodes are bootstrap

544 support values with a percentage based on 1000 replicates. Tn-Tetraodon nigroviridis, On-

545 Oreochromis niloticus, Po-P. olivaceus, Tr-T. rubripes, Pf-P. formosa, Ga-G. aculeatus. Ol-

546 Oryzias latipes, Cs-Cynoglossus semilaevis.

547

548 Figure 4 Chromosome synteny analysis of teleost GATA2 paralogs. Dotplots of the human

549 GATA2 gene region on human chr3 show double conserved synteny to the two GATA2 paralogs

550 in zebrafish on chromosomes Dre6 (GATA2b) and Dre11 (GATA2a).

551

552 Figure 5 Chromosomal segments showing the conserved syntenic blocks containing GATA2a and

553 GATA2b in teleosts. The genes are represented by colored pentagons, and the gene names are

554 indicated on top. Color pentagons indicate the same gene in different species and its respective

555 genomic position in relation to several other genes. The pentagon's direction indicates the gene

556 direction compared with the reference gene. The empty spaces indicate a region with other genes

557 or the absence of the gene in the genome. The blank pentagons indicate conserved genes between

558 GATA2a and GATA2b.

559

560 Figure 6 Expression of GATA2a (A) and GATA2b (B) in tongue sole organs relative of RPL17.

561 Data are shown as mean $\pm \operatorname{SEM}(n=3)$. Values with asterisks indicate statistical significance $(\mathrm{P}<$

562 0.05). B: brain; H: heart; I: intestine; K: kidney; L: liver; S: spleen; G: gonad. 
SUPPLEMENTAL INFORMATION

\section{Supplemental Figure Legends}

565

566

567

568

569

570

571

572

573

574

575

576

577

578

579

580

581

582

Figure S1 Multiple sequence alignment of the deduced GATA2a and GATA2b protein sequences.

Figure S2 Phylogenetic relationships, exon-intron structure, and motif structures of GATA2 genes. (A) ML phylogenetic tree and exon-intron structures of the GATA2 genes. Box: exon; lines: introns. The lengths of boxes and lines are scaled based on gene length. (B) MEME motif search results. Conserved motifs are indicated in numbered color boxes.

Figure S3 Phylogenetic tree of teleost GATA2 genes used in PAML analysis. (A) Phylogenetic tree constructed based on GATA2a sequences by using MrBayes with the TPM2uf + G model to assess selection pressure; $\mathrm{MCMC}=200,000$. (B) Phylogeny for site model constructed based on GATA2b sequences by using MrBayes with the TIM2+I model $(\mathrm{MCMC}=200,000)$.

Figure S4 Relative expression of GATA2a (A) and GATA2b (B) in female and male organs. Data are shown as mean $\pm \operatorname{SEM}(n=3)$. Values with different superscripts indicate statistical significance $(P<0.05)$

\section{Supplemental Tables}

Table S1 Database ID of the sequences used in this study.

Table S2 Primers used for qRT-PCR. 


\section{Supplemental Seq file}

584 Supplemental seq file.txt 
Figure 1 (on next page)

Phylogenetic analyses of vertebrate GATA gene family.

Phylogenetic tree constructed using MrBayes with the TPMIuf $+\mathrm{I}+\mathrm{G}$ model; MCMC $=400,000$ generations. On-Oreochromis niloticus, Cs-Cynoglossus semilaevis, Tn-Tetraodon nigroviridis, Mm-Mus musculus, Gg-Gallus gallus; Hs-Homo sapiens; Nv-Nematostella vectensis. 


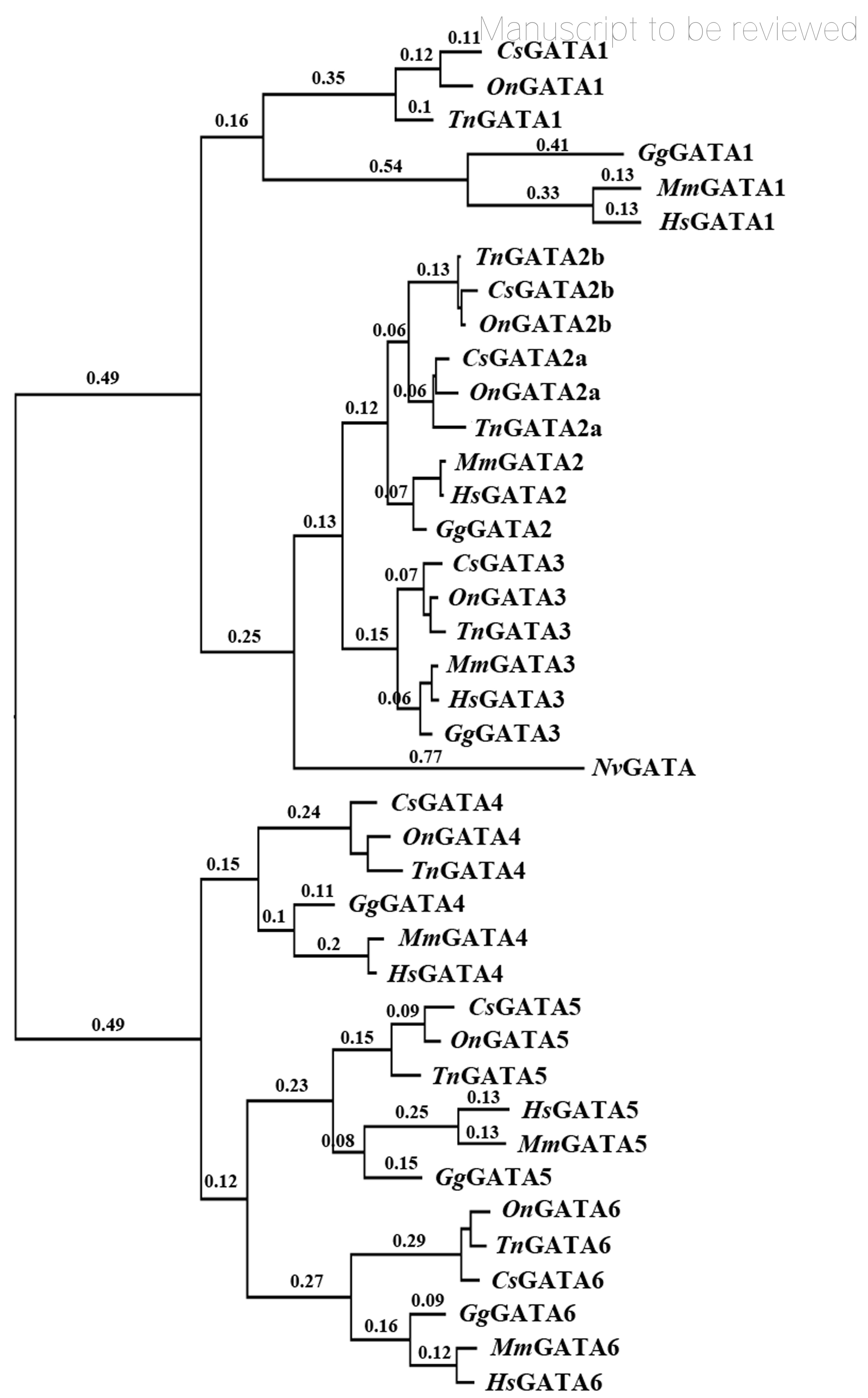


Figure 2 (on next page)

Partial multiple sequence alignment of the deduced GATA2a and GATA2b protein sequences.

In teleosts, two conserved zinc finger motifs were found in GATA2a and GATA2b (underlined sequences). Two GATA2b-specific mutations were identified in zinc finger motifs (star-shaped site). The proline site (arrowhead) in the N-terminal zinc finger motif had undergone positive selection. 


\section{*}

CsGATA2a GNGDPVCNACGL EGRECVNCGATSTPLWRRDGTGHYLCNACGLYHKMNGQNRPLIKPKRRLSAARRAGTCCANCOTTTTTLWRRNGNGDPVCNACGLY 
Figure 3 (on next page)

Phylogenetic analysis of teleost GATA2 .

(A) Phylogenetic tree constructed based on GATA2a and GATA2b in teleosts by using MrBayes with the TIM3+I+G model; $M C M C=400,000$. (B) Maximum likelihood phylogenetic tree constructed using phyML with the TIM3+I+G model. PhyML was run for 200 replications. Po-P.olivaceus, Tr-T. rubripes, Pf-P. formosa, Ga-G. aculeatus. 

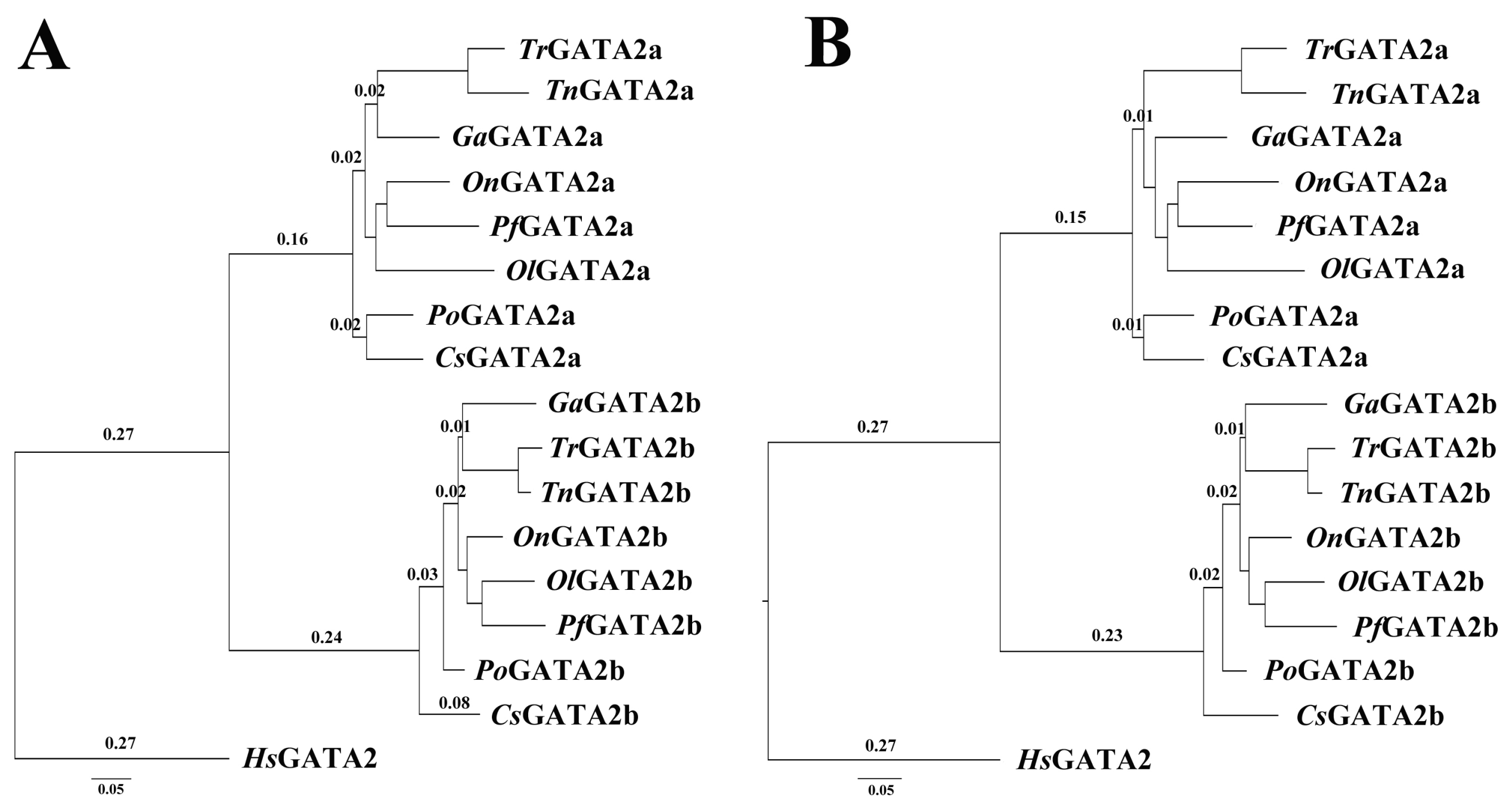


\section{Figure 4 (on next page)}

Chromosome synteny analysis of teleost GATA2 paralogs.

Dotplots of the human GATA2 gene region on human chr3 show double conserved synteny to the two GATA2 paralogs in zebrafish on chromosomes Dre6 (GATA2b) and Dre11 (GATA2a). 


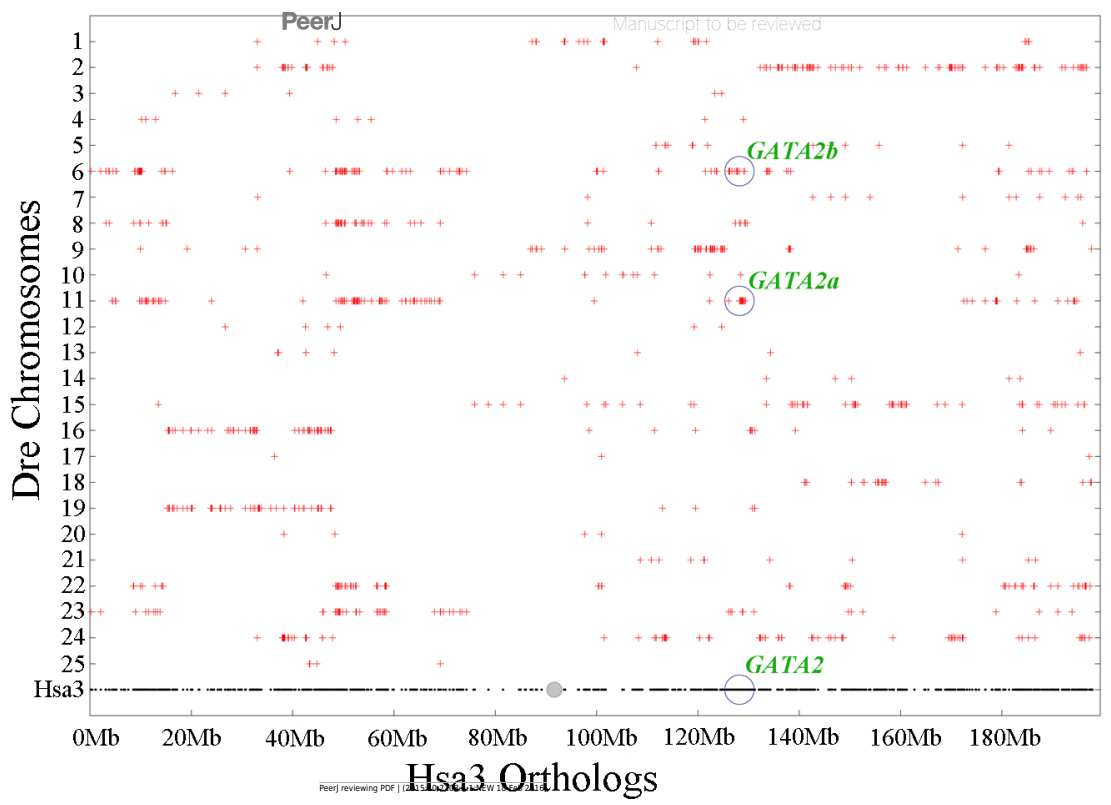


Figure $\mathbf{5}$ (on next page)

Chromosomal segments showing the conserved syntenic blocks containing GATA2a and GATA2b in teleosts.

The genes are represented by colored pentagons, and the gene names are indicated on top.

Color pentagons indicate the same gene in different species and its respective genomic position in relation to several other genes. The pentagon's direction indicates the gene direction compared with the reference gene. The empty spaces indicate a region with other genes or the absence of the gene in the genome. The blank pentagons indicate conserved genes between GATA2a and GATA2b. 
A

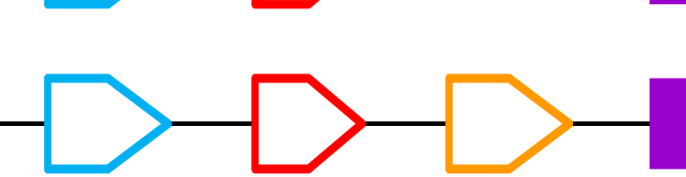

1

$\square-\square-$

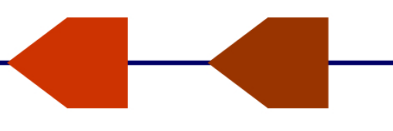

T. nigroviridis

B

mia40 sec61al Ip6k2 gar22 cidec uqcrcl hmces rab7a rpn1 GATA2b ITIH6 ITIH3 sfmbt1 prkcdb fgd5b uba3 tmf1 eogt fam19a4b fam19a1 suclg2

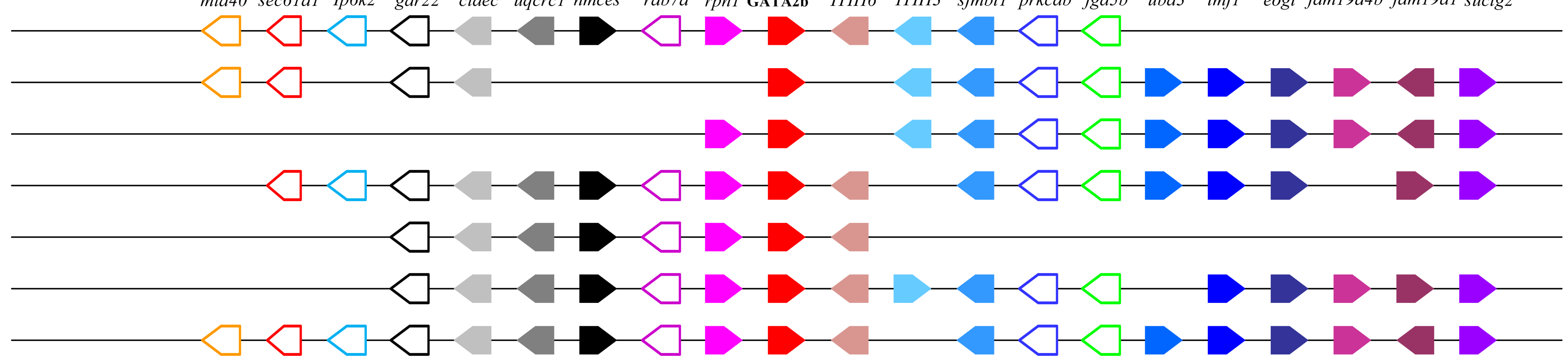

P. formosa

$X$. maculatus

O. latipes

O. niloticus

T. rubripes

T. nigroviridis

G. aculeatus 
Figure 6 (on next page)

Relative expression levels of GATA2a and GATA2b in tongue sole tissues.

(A) Relative expression level of GATA2a in males and females. (B) Relative expression level of GATA2b in males and females. B: brain; $\mathrm{H}$ : heart; I: intestine; $\mathrm{K}$ : kidney; L: liver; O: ovary; T: testis; S: spleen. 


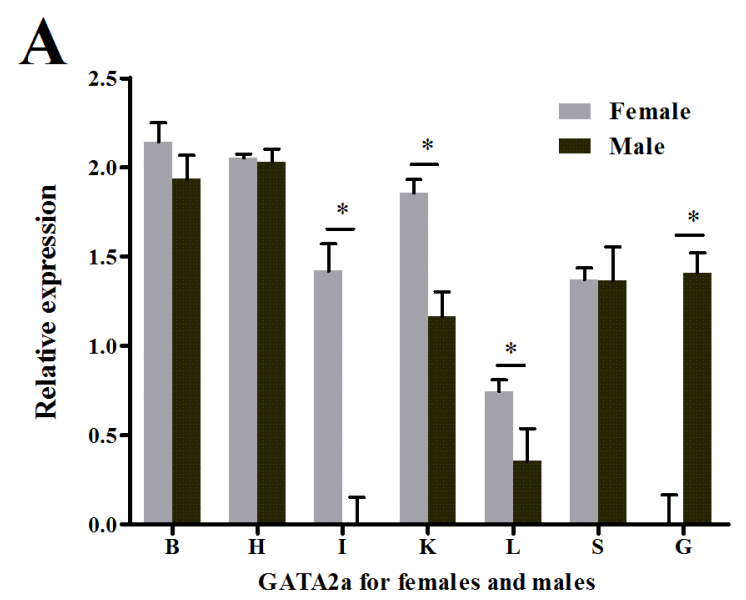

B

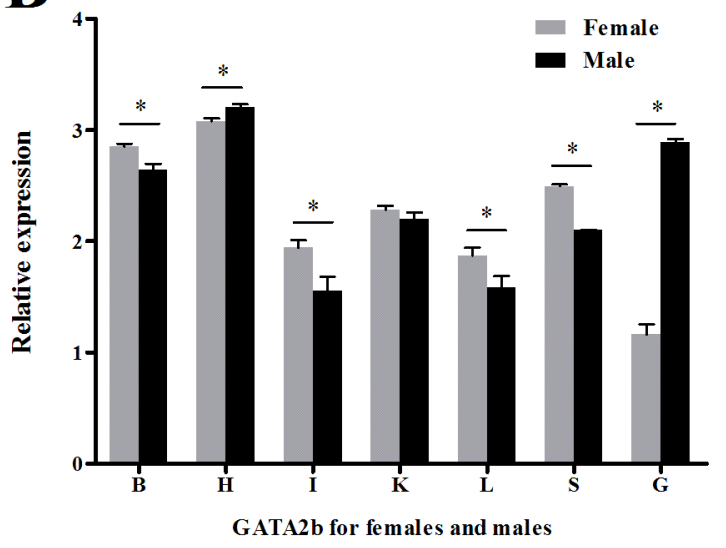




\section{Table 1 (on next page)}

Results of sites model analyses on the teleost GATA2 Bayesian gene tree. 
1 Table 1 Results of sites model analyses on the teleost GATA2 Bayesian gene tree.

\begin{tabular}{|c|c|c|c|c|c|c|c|c|c|}
\hline Tree & $\begin{array}{c}\text { Mod } \\
\text { el }\end{array}$ & $\ln L$ & $\kappa$ & Null & LRT & $\mathrm{df}$ & $P$-value & site & BEB \\
\hline \multirow{9}{*}{$\begin{array}{c}\text { GATA2 } \\
\text { a }\end{array}$} & M0 & -4832.177 & 2.463 & NA & & & & & \\
\hline & & & & & & & & & \\
\hline & M1a & -4775.943 & 2.642 & NA & & & & & \\
\hline & $\mathrm{M} 2 \mathrm{a}$ & -4775.943 & 2.642 & M1a & 0 & 2 & 1.000 & & \\
\hline & M3 & -4732.818 & 2.523 & M0 & 198.718 & 4 & 0.000 & & \\
\hline & M7 & -4733.231 & 2.524 & NA & & & & & \\
\hline & M8a & -4735.152 & 2.540 & NA & & & & & \\
\hline & M8 & -4733.231 & 2.524 & M7 & 0 & 2 & 1.000 & & \\
\hline & & & & M8a & 3.842 & 1 & 0.050 & & \\
\hline \multirow{8}{*}{$\begin{array}{c}\text { GATA2 } \\
\text { b }\end{array}$} & M0 & -4083.986 & 2.472 & NA & & & & & \\
\hline & M1a & -4053.831 & 2.560 & NA & & & & & \\
\hline & M2a & -4053.831 & 2.560 & M1a & 0 & 2 & 1.000 & & \\
\hline & M3 & -4034.159 & 2.488 & M0 & 99.654 & 4 & 0.000 & & \\
\hline & M7 & -4034.060 & 2.485 & NA & & & & & \\
\hline & M8a & -4037.538 & 2.506 & NA & & & & & \\
\hline & M8 & -4030.944 & 2.490 & M7 & 6.232 & 2 & 0.044 & $356(\mathrm{P})$ & $0.95^{*}$ \\
\hline & & & & M8a & 13.188 & 1 & 0.00028 & & \\
\hline
\end{tabular}

2 Note: Abbreviations: $\ln L$, ln likelihood; $\kappa$, transition/transversion ratio; df, degrees of freedom; NA, not applicable 
